\title{
Reincorporación al trabajo en el contexto de la pandemia de COVID-19 en sectores de industria y construcción en Navarra (España)
}

Return to work in the context of the COVID-19

pandemic in the industrial and construction sectors in Navarre (Spain)

Iñaki Moreno-Sueskun

Jorge Alberto Díaz-González ${ }^{1}$

Asier Acuña Juanbeltz ${ }^{1}$

Aingeru Pérez-Murillo ${ }^{1}$

Ana Garasa Jiménez ${ }^{1}$

Virginia García-Osés ${ }^{1}$

Estrella Extramiana Cameno ${ }^{1}$

'Servicio de Salud Laboral, Instituto de Salud Pública y Laboral de Navarra, Pamplona, España.

Fechas · Dates

Recibido: 2020.06 .23

Aceptado: 2020.10.14

Publicado: 2020.11.04

\section{Correspondencia $\cdot$ Corresponding Author}

Iñaki Moreno-Sueskun

imorenos@navarra.es 


\section{Resumen}

Objetivo: Analizar el grado de cumplimiento de las medidas preventivas dictadas por la Autoridad Sanitaria frente a la Covid-19 durante las fases de desescalada en empresas del sector industrial y de construcción en Navarra.

Métodos: Se elaboraron cuestionarios a partir de las medidas establecidas para verificar in situ el grado de aplicación. El personal técnico de prevención del Servicio de Salud Laboral (SSL) del Instituto de Salud Pública y Laboral de Navarra y de los servicios de prevención ajenos (SPA) visitaron las empresas para asesorar, comprobar y requerir, en su caso, el cumplimiento de las medidas dictadas.

Resultados: El grado de cumplimiento de las medidas es mayor en industria que en construcción. Se han generalizado las prácticas de limpieza, los recursos higiénicos y la disposición de elementos de protección respiratoria. La aplicación de medidas necesarias en los lugares comunes (baños, comedores) es menos frecuente. Hay carencia de cartelería informativa y de constancia escrita de las instrucciones preventivas. Se observan diferencias de varios resultados entre la labor desarrollada por los SPA y por el SSL.

Conclusiones: Se han detectado debilidades en la consecución de espacios seguros en el trabajo en los sectores de la construcción e industria durante la fase de desescalada en el contexto de la pandemia de la Covid-19. Debe ser reconsiderado el papel de la salud pública en las empresas y la integración de los servicios de prevención en las políticas públicas frente a los riesgos laborales.

Palabras clave: COVID-19; riesgos laborales; reincorporación laboral; medidas preventivas.

\section{Abstract}

Objective: To analyse the compliance of the preventive instructions formulated by the Health Authority against COVID-19 for the de-escalation phase among the industrial and construction sectors in Navarre.

Methods: On the basis of those preventive instructions, questionnaires were drawn up to verify their implementation on site. The occupational risk prevention technicians of the Occupational Health Service (OHS) of the Institute of Public and Work Health of Navarre and those of the External Prevention Services (EPS), under the executive coordination of the $\mathrm{OHS}$, visited the enterprises to advise, verify and require, if necessary, compliance with the instructions dictated.

Results: The compliance of the preventive instructions is higher in the industrial sector than in the construction sector. Cleaning practices, hygienic resources and the provision of respiratory protection equipment are widespread. The application of necessary measures in common places (restrooms, dining rooms) are less frequent. There is a lack of informative posters and written corroboration of the preventive instructions. The difference of several results between the OHS and the EPS technicians is remarkable.

Conclusions: Weaknesses have been identified in achieving safe workplaces in the construction and industrial sectors during the de-escalation phase in the context of the Covid-19 pandemic. The role of Public Health in the enterprises and the integration of the prevention services in public policies against occupational risks must be reconsidered.

Keywords: COVID-19; occupational risk; return to work; preventive measures. 


\section{Introducción}

La enfermedad Covid-19 se ha caracterizado como un problema de salud pública por la Organización Mundial de la Salud (OMS) y una significativa proporción de casos está relacionada con exposiciones laborales ${ }^{(1)}$. Las tareas que supongan proximidad interpersonal presentan un mayor riesgo de contagio(2). El Gobierno central decretó el estado de alarma en España el 14 de marzo de 2020, estableciendo el confinamiento general de la población con la paralización de toda actividad económica no esencial. El 10 de abril se autorizó la vuelta al trabajo de los sectores industrial y de construcción en la llamada "desescalada". Con ello, las empresas de ambos sectores, excepto las obras menores con concurrencia de personas ajenas a la obra, podían reanudar su actividad laboral.

Este estudio analiza el cumplimiento de las medidas preventivas dictadas por la Autoridad Sanitaria frente a la Covid-19 durante la fase de desescalada en las empresas industriales y de construcción de Navarra.

\section{Métodos}

\section{Población a estudio}

En Navarra, en el periodo de realización de este estudio, existían 2.017 empresas industriales y 1.621 constructivas que emplean a 73.411 personas asalariadas (Registro de Tesorería General de la Seguridad Social). En construcción, considerando los datos por apertura de centro de trabajo de obras notificadas al Gobierno de Navarra (GN) en los 5 últimos años, se estima una media anual de 3.000 obras con planes de seguridad y salud, que constituyen la población de este estudio.

\section{Diseño}

El GN asignó al SSL del Instituto de Salud Pública y Laboral de Navarra (ISPLN) la tarea de acompañar la vuelta al trabajo segura de los sectores de industria y construcción, en la desescalada laboral del confinamiento.

El SSL, en la segunda semana de abril de 2020, elaboró sendos borradores de medidas preventivas a implantar en empresas y obras. Fueron consultados con la autoridad laboral autonómica y con los agentes empresariales y sindicales del Consejo Navarro de Salud Laboral (órgano consultivo tripartito), integrándose sus aportaciones. En la segunda quincena de abril, las medidas definitivas ${ }^{(3,4)}$ fueron remitidas a todas las empresas a través de la Asociación de Mutuas de Accidentes de Trabajo (Amat) y asociaciones de empresarios/as y del trabajo autónomo.

Teniendo en cuenta dichas medidas, las unidades correspondientes de la Sección técnica del SSL elaboraron cuestionarios de verificación (en adelante cuestionarios), de respuesta binaria, para registrar la comprobación de la implantación de las medidas. Estos cuestionarios se acompañaban de criterios de cumplimentación de los mismos, así como de un manual de visitas para la realización del tra- 
bajo de campo. Para comprobar la aplicación de las medidas se estableció un programa de visitas (PV) a los centros de trabajo.

El GN encargó al SSL, mediante Decreto-ley Foral(5), el ejercicio de la coordinación ejecutiva (dirección y gestión) de los SPA. La tercera semana de abril se envió un correo electrónico a todos los SPA concernidos, solicitando la remisión de los datos sobre sus recursos humanos y técnicos, así como de las empresas de industria y construcción con las que tuvieran concertadas las disciplinas preventivas de higiene industrial y/o medicina del trabajo en Navarra. Por las limitaciones propias del contexto de pandemia, no se formó presencialmente al personal de los SPA sobre los manuales de instrucciones de cumplimentación de los cuestionarios previamente a su visita a centros de trabajo. Sin perjuicio de ello, todas sus dudas fueron resueltas telemáticamente.

El trabajo de campo se desarrolló entre el 23 de abril y el 5 de junio de 2020. Los cuestionarios eran cumplimentados por el personal técnico del SSL y de los SPA tras verificación objetiva, comprobación documental y entrevistas con los diversos agentes de la empresa.

Las visitas a los centros de trabajo fueran realizadas sin previo aviso. En las pequeñas empresas industriales de menos de 10 personas, el tiempo de cumplimentación del cuestionario fue aproximadamente de dos horas, mientras en las medianas y grandes se requirieron entre cinco y seis horas. Estos tiempos fueron de 15 minutos, en edificación unifamiliar, progresando hasta los 75 minutos en función de la mayor dimensión de obra.

En este programa de visitas han participado 15 técnicos del SSL y 77 de los SPA.

El SSL visitó inicialmente empresas de la comarca de Pamplona a modo de estudio piloto, y, posteriormente, aquellas que según las bases de datos del ISPLN no disponían de SPRL ( $\mathrm{n}=132)$. Visitó empresas de entre 2 y 99 trabajadores. Cada SPRL debía visitar sus empresas atendidas. En el estudio piloto del sector de construcción el personal del SSL visitó de forma oportunista las mayores obras de la comarca de Pamplona y Tudela, para pasar posteriormente a tutelar la actuación de los SPA.

Al llegar a la empresa u obra, se reclamaba la presencia del responsable máximo de la misma y de representantes del personal en caso de haberlos, preferentemente delgadas/os de prevención. Tras comprobar documentalmente registros, procedimientos de limpieza, protocolos de uso de zonas comunes etc., se recorrían todas las áreas de la empresa, en especial los lugares de producción y los espacios comunes (comedor, baños, vestuarios, zonas de descanso, y otras). Por último, se contrastaban determinadas respuestas dadas por la empresa con la representación del personal.

Durante la visita, en caso de incumplimiento de cualquiera de las medidas más importantes (presencia de personal indispensable, distancias interpersonales, productos de limpieza e higiene, zonas comunes, formación e información, protección respiratoria) se transmitía verbalmente la obligación de implantarlas de manera inmediata. 
Tras la visita, se enviaba correo electrónico a la empresa instando a la resolución de los incumplimientos detectados. En construcción, se remitía además el referido mensaje electrónico al coordinador/a de seguridad y salud de la obra. En 48 horas la empresa debía remitir al SSL evidencias (documentales, fotográficas) de la aplicación de las medidas exigidas.

En este PV han participado exclusivamente SPA. Se descartó incluir a los servicios de prevención propios, presumiendo que atendían las necesidades preventivas de sus empresas. Los servicios mancomunados, en el momento de realización de este estudio, no habían visitado empresas.

Los cuestionarios cumplimentados por el personal técnico de los SPA debían ser remitidos al SSL en el plazo de dos días tras la visita.

Se habilitaron formularios de Google Drive para el volcado de los datos por el personal técnico. Al objeto de garantizar la confidencialidad de la información, se asignó un código a cada una de sus empresas y obras visitadas. Los SPA remitían semanalmente al SSL las razones sociales de las empresas y obras visitadas, junto a sus códigos correspondientes, a una única dirección electrónica.

Se analizaron los resultados calculando los porcentajes de empresas en las que, procediendo la aplicación de la medida preventiva (cada item del cuestionario), se cumple de forma considerable, según criterio del/la técnico cumplimentador.

\section{Resultados}

Se visitaron 871 empresas industriales, de las cuales 212 se encontraron cerradas o ilocalizables. De las 659 restantes, el 54,8\% fueron visitadas por el SSL y el 45,2\% por los SPA.

En la industria de Navarra actúan 23 SPA. De ellos, 2 no han prestado colaboración con este PV, y 3 de ellos cuando se reclamó su intervención se encontraban en situación de ERTE, total o parcial. A día 8 de junio, se disponía de información del $32,7 \%$ (659/2017) de las empresas industriales de Navarra con actividad, considerando que todas las no visitadas estaban activas. De las 361 empresas visitadas por el SSL, 36 no cuentan con modalidad preventiva alguna (10\%).

En construcción, de los 19 SPA que atienden este sector, 5 no han colaborado, y 1 de ellos, de gran dimensión, ha visitado una empresa de construcción tras dos meses de inicio del programa. Se visitaron 114 obras en las que trabajan 183 empresas en total: 114 contratas (62\%), 60 subcontratas (32\%), 7 autónomos (4\%), 2 $(0,1 \%)$ valores perdidos.

\section{Construcción}

Se observa en laTabla 1 y en la Tabla 2 los ítems del cuestionario para las obras. Se presentan los resultados del personal de SPA y del SSL Se expone el número de empresas de las obras de construcción en las que procede la medida preven- 
tiva (N), que varía según tipo y fase de la obra, y el porcentaje de empresas que la cumple (\%).

Tabla 1. Proporción de cumplimiento del sector de construcción de las medidas preventivas organizativas en relación con la organización, zonas comunes y limpiezas emanadas desde la Autoridad Sanitaria frente a la Covid-19 en obra de construcción en la desescalada laboral. Navarra. Abril - junio 2020.

\begin{tabular}{|c|c|c|c|c|c|}
\hline & & SPA & & SSL & \\
\hline $\begin{array}{l}\text { Item } \\
\text { (IV) a }\end{array}$ & Medida preventiva & $\mathbf{n}$ & $\%$ & $\mathbf{n}$ & $\%$ \\
\hline 1 & $\begin{array}{l}\text { La entrada y salida de la obra se realiza } \\
\text { de forma escalonada }\end{array}$ & 110 & 100,0 & 21 & 95,2 \\
\hline 2 & $\begin{array}{l}\text { Durante la entrada, se toman medidas } \\
\text { para garantizar que los trabajadores } \\
\text { no esperan juntos sino respetando la } \\
\text { distancia de } 2 \text { metros }\end{array}$ & 110 & 98,2 & 20 & 85,0 \\
\hline 3 & $\begin{array}{l}\text { Existen instrucciones por escrito de } \\
\text { esa entrada y salida }\end{array}$ & 101 & 60,4 & 21 & 28,6 \\
\hline 4 & $\begin{array}{l}\text { Se planifican diariamente las tareas } \\
\text { para establecer aquellas en las que } \\
\text { deben utilizarse EPI }\end{array}$ & 111 & 95,5 & 21 & 33,3 \\
\hline 5 & $\begin{array}{l}\text { Se organizan turnos en dichas tareas } \\
\text { para que los trabajadores no tengan } \\
\text { que estar más de } 4 \text { horas (como } \\
\text { máximo) con protección respiratoria } \\
\text { al día }\end{array}$ & 101 & 90,1 & 20 & 25,0 \\
\hline 6 & $\begin{array}{l}\text { Existe cartelería con las } \\
\text { recomendaciones contra el Covid-19 }\end{array}$ & 109 & 66,1 & 15 & 53,3 \\
\hline 7 & Existen vestuarios habilitados & 94 & 77,7 & 15 & 46,7 \\
\hline 8 & $\begin{array}{l}\text { Se han dado instrucciones por } \\
\text { escrito de cómo utilizar los vestuarios } \\
\text { garantizando la distancia de }>\text { de } 2 \mathrm{~m}\end{array}$ & 69 & 69,6 & 15 & 46,7 \\
\hline 9 & $\begin{array}{l}\text { Se ha establecido el número máximo } \\
\text { de usuarios simultáneamente de } \\
\text { las zonas comunes en función de } \\
\text { su tamaño, para garantizar que se } \\
\text { respetan las distancias }\end{array}$ & 66 & 75,8 & 15 & 46,7 \\
\hline 10 & $\begin{array}{l}\text { Se ha realizado un protocolo de } \\
\text { limpieza y desinfección diario de los } \\
\text { vestuarios }\end{array}$ & 66 & 65,2 & 9 & 22,2 \\
\hline 11 & $\begin{array}{l}\text { Se ha instaurado registro diario de } \\
\text { limpieza del vestuario (nombre, fecha, } \\
\text { hora, firma) }\end{array}$ & 58 & 32,8 & 10 & 30,0 \\
\hline 12 & Existen baños con lavabos habilitados & 101 & 88,1 & 15 & 46,7 \\
\hline 13 & $\begin{array}{l}\text { Se han dado instrucciones por } \\
\text { escrito de cómo utilizar los baños } \\
\text { garantizando la limpieza y desinfección } \\
\text { tras el uso por cada trabajador }\end{array}$ & 83 & 61,4 & 13 & 53,8 \\
\hline
\end{tabular}




\begin{tabular}{|c|c|c|c|c|c|}
\hline & & SPA & & SSL & \\
\hline $\begin{array}{l}\text { Item } \\
\text { (IV) a }\end{array}$ & Medida preventiva & $\mathbf{n}$ & $\%$ & n & $\%$ \\
\hline 14 & $\begin{array}{l}\text { Se dispone en los lavabos de jabón, } \\
\text { toallitas de papel y papelera }\end{array}$ & 83 & 71,1 & 8 & 87,5 \\
\hline 15 & $\begin{array}{l}\text { Se dispone en los lavabos de gel } \\
\text { hidroalcohólico }\end{array}$ & 85 & 78,8 & 7 & 71,4 \\
\hline 16 & $\begin{array}{l}\text { Se dispone en los lavabos de cartelería } \\
\text { explicativa de cómo lavarse las manos }\end{array}$ & 84 & 59,5 & 7 & 42,9 \\
\hline 17 & $\begin{array}{l}\text { Se ha instaurado registro diario de } \\
\text { limpieza de los baños (nombre, fecha, } \\
\text { hora, firma) }\end{array}$ & 75 & 34,7 & 7 & 42,9 \\
\hline 18 & Existen comedores habilitados & 78 & 67,9 & 14 & 21,4 \\
\hline 19 & $\begin{array}{l}\text { En el comedor se dispone de productos } \\
\text { para la higiene personal (jabón / agua } \\
\text { corriente /papel / papelera) }\end{array}$ & 50 & 68,0 & 4 & 25,0 \\
\hline 20 & $\begin{array}{l}\text { En el comedor se dispone de } \\
\text { productos para que cada trabajador } \\
\text { pueda desinfectar la zona en la que } \\
\text { ha comido previamente a su uso por } \\
\text { otros trabajadores o se han establecido } \\
\text { turnos fijos con limpieza intermedio } \\
\text { por un tercero }\end{array}$ & 48 & 64,6 & 4 & 25,0 \\
\hline 21 & $\begin{array}{l}\text { Se han dado instrucciones por escrito } \\
\text { de cómo utilizar los comedores tanto } \\
\text { por turnos como para su limpieza tras } \\
\text { el uso }\end{array}$ & 56 & 62,5 & 7 & 0,0 \\
\hline 22 & $\begin{array}{l}\text { Se ha establecido un procedimiento de } \\
\text { limpieza y desinfección de zonas de } \\
\text { uso común }\end{array}$ & 49 & 67,3 & 40 & 67,5 \\
\hline 23 & $\begin{array}{l}\text { Se ha instaurado registro diario de } \\
\text { limpieza de zonas comunes (nombre, } \\
\text { fecha, hora, firma) }\end{array}$ & 46 & 41,3 & 4 & 0,0 \\
\hline \multicolumn{2}{|c|}{ Total de empresas de las obras visitadas } & 125 & 100 & 58 & 100 \\
\hline
\end{tabular}

a Ítem del cuestionario a valorar. SPA: Servicio de Prevención Ajeno SSL: Servicio de Salud Laboral del ISPLN 
Tabla 2. Proporción de cumplimiento del sector de construcción de las medidas preventivas en relación con los equipos de trabajo, equipos, información y formación, conductas seguras frente a la Covid-19 emanadas desde la Autoridad Sanitaria en la desescalada laboral en Navarra. Abril - junio 2020.

\begin{tabular}{|c|c|c|c|c|c|}
\hline & & SPA & & SSL & \\
\hline $\begin{array}{l}\text { Ítem } \\
\text { (IV) }\end{array}$ & Medida preventiva & $\mathbf{n}$ & $\%$ & $\mathbf{n}$ & $\%$ \\
\hline 24 & $\begin{array}{l}\text { Se ha establecido un procedimiento } \\
\text { para la recepción de materiales }\end{array}$ & 87 & 71,3 & 54 & 13,0 \\
\hline 25 & $\begin{array}{l}\text { Se ha establecido un procedimiento de } \\
\text { limpieza y desinfección de máquinas } \\
\text { móviles y vehículo (quién + cómo + } \\
\text { cuándo) }\end{array}$ & 95 & 73,7 & 49 & 18,4 \\
\hline 26 & $\begin{array}{l}\text { Se dispone en la obra de un } \\
\text { procedimiento de limpieza y } \\
\text { desinfección de equipos de trabajo / } \\
\text { herramientas (quién, cómo, cuándo) }\end{array}$ & 105 & 56,2 & 58 & 22,4 \\
\hline 27 & $\begin{array}{l}\text { Existe zona específica para depositar la } \\
\text { herramienta limpia }\end{array}$ & 94 & 57,4 & 56 & 19,6 \\
\hline 28 & $\begin{array}{l}\text { Se prioriza el uso por un único } \\
\text { trabajador de herramientas / máquinas }\end{array}$ & 115 & 89,6 & 22 & 86,4 \\
\hline 29 & $\begin{array}{l}\text { Se ha informado a los trabajadores de } \\
\text { que en caso de compartir herramienta, } \\
\text { debe desinfectarse tras o previamente } \\
\text { a cada uso }\end{array}$ & 112 & 92,0 & 22 & 13,6 \\
\hline 30 & $\begin{array}{l}\text { Se garantiza que la distancia de } \\
\text { separación entre los trabajadores es } \\
\text { mayor a } 2 \text { metros }\end{array}$ & 113 & 90,3 & 22 & 72,7 \\
\hline 31 & $\begin{array}{l}\text { Se ha dispuesto de barreras físicas en } \\
\text { alguna zona de la obra }\end{array}$ & 69 & 39,1 & 46 & 0,0 \\
\hline 32 & $\begin{array}{l}\text { Existen tareas que exigen la utilización } \\
\text { de mascarillas y se están empleando }\end{array}$ & 122 & 93,4 & 56 & 71,4 \\
\hline 33 & En tal caso, ¿se están empleando? & 114 & 100,0 & 19 & 31,6 \\
\hline 34 & $\begin{array}{l}\text { Las mascarillas utilizadas son FFP2 o } \\
\text { KN } 95 \text { o similar (revisar en el almacén) }\end{array}$ & 117 & 77,8 & 55 & 21,8 \\
\hline 35 & $\begin{array}{l}\text { Los trabajadores utilizan las mascarillas } \\
\text { correctamente: colocación correcta, no } \\
\text { se quitan para hablar, no se reutilizan } \\
\text { tras usos, no se dejan colgadas o sobre } \\
\text { el casco }\end{array}$ & 122 & 95,1 & 17 & 23,5 \\
\hline 36 & $\begin{array}{l}\text { Se utilizan guantes de protección frente } \\
\text { a virus o microorganismos EN 374-5 }\end{array}$ & 110 & 53,6 & 56 & 10,7 \\
\hline 37 & $\begin{array}{l}\text { Se colocan y retiran los EPI } \\
\text { correctamente (desinfección previa, } \\
\text { desecho...) }\end{array}$ & 121 & 90,1 & 18 & 16,7 \\
\hline 38 & $\begin{array}{l}\text { Se dispone en los vestuarios de gel } \\
\text { hidroalcohólico para la limpieza entre } \\
\text { cambio de ropa }\end{array}$ & 97 & 77,3 & 11 & 36,4 \\
\hline
\end{tabular}




\begin{tabular}{|c|c|c|c|c|c|}
\hline & & SPA & & SSL & \\
\hline $\begin{array}{l}\text { Ítem } \\
\text { (IV) }\end{array}$ & Medida preventiva & $\mathbf{n}$ & $\%$ & n & $\%$ \\
\hline 39 & $\begin{array}{l}\text { Se dispone de productos de } \\
\text { desinfección: Lejía / Alcohol / Listado } \\
\text { legal de viricidas }\end{array}$ & 118 & 84,7 & 51 & 72,5 \\
\hline 40 & $\begin{array}{l}\text { Se ha informado verbalmente de los } \\
\text { síntomas y cómo actuar en caso de su } \\
\text { aparición }\end{array}$ & 121 & 98,3 & 58 & 79,3 \\
\hline 41 & $\begin{array}{l}\text { Se ha informado por escrito de los } \\
\text { síntomas y cómo actuar en caso de su } \\
\text { aparición }\end{array}$ & 125 & 77,6 & 58 & 53,4 \\
\hline 42 & $\begin{array}{l}\text { Se ha informado verbalmente de } \\
\text { las medidas de prevención eficaces: } \\
\text { aislamiento e higiene personal }\end{array}$ & 120 & 100,0 & 58 & 84,5 \\
\hline 43 & $\begin{array}{l}\text { Se ha informado por escrito de las } \\
\text { medidas de prevención eficaces: } \\
\text { aislamiento e higiene personal }\end{array}$ & 125 & 78,4 & 58 & 46,6 \\
\hline 44 & $\begin{array}{l}\text { Se ha informado de manera práctica de } \\
\text { las medidas a adoptar en la obra frente } \\
\text { a Covid19 }\end{array}$ & 125 & 92,8 & 22 & 45,5 \\
\hline 45 & $\begin{array}{l}\text { Conocen dónde se encuentran los } \\
\text { productos de higiene personal }\end{array}$ & 123 & 98,4 & 19 & 78,9 \\
\hline 46 & Saben cómo deben lavarse las manos & 125 & 99,2 & 22 & 72,7 \\
\hline 47 & $\begin{array}{l}\text { Se les ha formado de manera práctica } \\
\text { en el uso de mascarilla y guantes }\end{array}$ & 124 & 82,3 & 58 & 31,0 \\
\hline \multicolumn{2}{|c|}{ Total de empresas de las obras visitadas } & 125 & 100.0 & 58 & 100.0 \\
\hline
\end{tabular}

a Ítem del cuestionario a valorar. SPA: Servicio de Prevención Ajeno SSL: Servicio de Salud Laboral del ISPLN

Se han visitado 114 obras, en las cuales generalmente coinciden diversas empresas. Según la actividad o tareas de la empresa en la obra puede proceder, o no, la aplicación de la medida.

En relación a las medidas, vemos en las tablas 1 y 2 que se han adoptado medidas para mantener la distancia de más de 2 metros a la entrada (item a verificar -IV, en adelante- 2) a la obra, pero se ha hecho en menor medida respecto al uso de espacios comunes (IV8, IV9). El cumplimiento de medidas organizativas para una entrada y salida adecuadas al trabajo (IV1) es general. Dependiendo del tipo de entidad informante, las medidas para favorecer el uso adecuado de los EPI (IV4, IV5), varía entre $1 / 3$ y 2/3 de las empresas. Lo mismo ocurre con las medidas sobre limpieza de lugares comunes, herramienta y sus procedimientos (IV10, IV11, IV17, IV20, IV21, IV22, IV23, IV27 e IV28). La disposición de productos de higiene personal (IV14, IV15, IV19, IV38, IV45, IV 46) es muy frecuente, pero no en comedores y vestuarios según el SSL. En cuanto a necesidad, disposición y uso correcto de EPI y otras protecciones el SSL valora muy críticamente la situación (IV31 a IV37, IV47) contrariamente a los SPA, destacando la baja frecuencia de uso correcto de los elementos protectores. Respecto a medidas de información y formación (IV6, IV16, IV29, IV40, IV41, IV42, IV43, IV44 e IV47) destaca la información dada 
sobre síntomas y medidas preventivas generales frente a la enfermedad. El aspecto práctico de la formación en el uso de EPI solo se ha realizado en el 30\% de las obras según el SSL, dato coherente con el generalizado uso incorrecto del mismo observado por su personal (IV35, IV37). Los SPA consideran que el cumplimiento de estas medidas es muy frecuente.

En todos los aspectos en los que el cuestionario se ha interesado por la constancia escrita (instrucciones, registros, procedimientos escritos, información gráfica, etc.) se observa una frecuencia bastante menor que la que se afirma realizar en lo referido a lavado de manos (V17), uso de comedores (V21), limpieza general (V23), información sobre síntomas (V41) o sobre medidas preventivas (V43).

\section{Industria}

En las 298 empresas visitadas por los SPA, el 71,5\% de los 14.639 trabajadores/ as estaba en activo; $y$, en las 358 visitadas por el SSL, el 73,4\% de los 7.173 trabajadores/as estaba activo.

Tabla 3. Proporción de cumplimiento del sector industrial de las medidas frente a la Covid 19 emanadas desde la Autoridad Sanitaria en la desescalada laboral en Navarra. Abril - junio 2020.

\begin{tabular}{|c|c|c|c|c|c|}
\hline & & SPA & & SSL & \\
\hline $\begin{array}{l}\text { Item } \\
\text { (IV) }\end{array}$ & Medida preventiva & $\mathbf{n}$ & $\%$ & $\mathbf{n}$ & $\%$ \\
\hline 1 & $\begin{array}{l}\text { Los trabajadores han participado en la } \\
\text { adopción de medidas frente al Covid-19 }\end{array}$ & 293 & 85,7 & 346 & 54,6 \\
\hline 2 & $\begin{array}{l}\text { Permanece en el centro el mínimo } \\
\text { personal posible }\end{array}$ & 277 & 96,8 & 324 & 79,9 \\
\hline 3 & $\begin{array}{l}\text { Se han prohibido las visitas no } \\
\text { imprescindibles }\end{array}$ & 289 & 96,5 & 348 & 93,1 \\
\hline 4 & $\begin{array}{l}\text { El personal de las contratas se guía por } \\
\text { los mismos criterios que el propio }\end{array}$ & 248 & 97,6 & 260 & 89,2 \\
\hline 5 & $\begin{array}{l}\text { Se han implantado medidas sobre } \\
\text { aspectos psicosociales }\end{array}$ & 278 & 61,2 & 329 & 41,0 \\
\hline 6 & $\begin{array}{l}\text { La entrada y salida al trabajo está } \\
\text { organizada (no aglomeraciones y se } \\
\text { mantienen las distancias) }\end{array}$ & 281 & 97,2 & 323 & 93,8 \\
\hline 7 & $\begin{array}{l}\text { En las zonas de trabajo se garantiza la } \\
\text { distancia interpersonal > } 2 \mathrm{~m} \text { o se han } \\
\text { instalado separaciones físicas }\end{array}$ & 294 & 92,2 & 355 & 87,6 \\
\hline 8 & $\begin{array}{l}\text { Planificación diaria de las tareas para } \\
\text { detectar aquellas con posibilidad de } \\
\text { distancia interpersonal }<2 m\end{array}$ & 248 & 90,7 & 185 & 72,4 \\
\hline 9 & $\begin{array}{l}\text { En zonas comunes se garantiza } \\
\text { distancia interpersonal }>2 \mathrm{~m} \text {, o se han } \\
\text { instalado separaciones físicas }\end{array}$ & 286 & 95,1 & 334 & 85,3 \\
\hline 10 & $\begin{array}{l}10 \text { El personal mantiene dichas } \\
\text { distancias (observación directa) }\end{array}$ & 294 & 89,1 & 239 & 89,1 \\
\hline
\end{tabular}




\begin{tabular}{|c|c|c|c|c|c|}
\hline & & SPA & & SSL & \\
\hline $\begin{array}{l}\text { Item } \\
\text { (IV) }\end{array}$ & Medida preventiva & $\mathbf{n}$ & $\%$ & $\mathbf{n}$ & $\%$ \\
\hline 11 & $\begin{array}{l}\text { Hay control de que se mantienen tales } \\
\text { distancias entre el personal }\end{array}$ & 277 & 79,1 & 208 & 44,7 \\
\hline 12 & $\begin{array}{l}\text { Medidas adoptadas para aumentar la } \\
\text { renovación del aire interior }\end{array}$ & 263 & 83,7 & 310 & 64,5 \\
\hline 13 & $\begin{array}{l}\text { Disponen productos para limpieza y } \\
\text { desinfección de superficies, materiales } \\
\text { y equipos de trabajo }\end{array}$ & 296 & 98,6 & 357 & 91,9 \\
\hline 14 & $\begin{array}{l}\text { Existen instrucciones específicas por } \\
\text { escrito para la limpieza y desinfección } \\
\text { a fondo }\end{array}$ & 291 & 70,4 & 351 & 46,7 \\
\hline 15 & $\begin{array}{l}\text { Se garantiza la limpieza y desinfección } \\
\text { de la zona y equipos de trabajo entre } \\
\text { cada turno o uso por parte del personal }\end{array}$ & 290 & 90,3 & 344 & 76,7 \\
\hline 16 & $\begin{array}{l}\text { Se garantiza la limpieza y desinfección } \\
\text { de las zonas comunes tras cada } \\
\text { "tanda" de uso }\end{array}$ & 288 & 85,8 & 349 & 80,2 \\
\hline 17 & $\begin{array}{l}\text { Se dispone de etanol o hidrogel para la } \\
\text { desinfección de manos }\end{array}$ & 297 & 98,3 & 246 & 93,1 \\
\hline 18 & $\begin{array}{l}\text { Los productos para desinfección de } \\
\text { manos son viricidas autorizados o } \\
\text { etanol al } 70 \% \text { mínimo }\end{array}$ & 283 & 100,0 & 185 & 100,0 \\
\hline 19 & $\begin{array}{l}\text { Se ha establecido el uso individual de } \\
\text { útiles y herramientas de trabajo o su } \\
\text { desinfección antes y tras cada uso }\end{array}$ & 288 & 94,8 & 287 & 80,5 \\
\hline 20 & $\begin{array}{l}\text { Se dispone de papeleras o } \\
\text { contenedores con tapa para desechar } \\
\text { mascarillas, guantes y/o pañuelos } \\
\text { desechables }\end{array}$ & 284 & 79,2 & 333 & 73,6 \\
\hline 21 & $\begin{array}{l}\text { Se ha formado o informado sobre las } \\
\text { medidas adoptadas en el centro de } \\
\text { trabajo }\end{array}$ & 295 & 96,6 & 358 & 84,1 \\
\hline 22 & $\begin{array}{l}\text { Se ha proporcionado formación } \\
\text { teórico-práctica en uso de EPI (si } \\
\text { procede) }\end{array}$ & 237 & 85,7 & 317 & 42,9 \\
\hline 23 & $\begin{array}{l}\text { Disponen de carteles relativos al } \\
\text { lavado de manos, prácticas higiénicas } \\
\text { respiratorias, etc. }\end{array}$ & 295 & 86,8 & 351 & 58,4 \\
\hline 24 & $\begin{array}{l}\text { Es necesario el uso de protección } \\
\text { respiratoria }\end{array}$ & 286 & 65,4 & 224 & 29,5 \\
\hline 25 & $\begin{array}{l}\text { Disponen de EPI de protección } \\
\text { respiratoria (mascarilla auto filtrante } \\
\text { FFP2 o superior) }\end{array}$ & 288 & 83,3 & 215 & 81,9 \\
\hline 26 & Disponen de mascarilla quirúrgica IIR & 268 & 62,7 & 122 & 54,1 \\
\hline 27 & $\begin{array}{l}\text { Disponen de otras mascarillas } \\
\text { quirúrgicas (No IIR) }\end{array}$ & 267 & 52,1 & 118 & 77,1 \\
\hline
\end{tabular}




\begin{tabular}{|c|c|c|c|c|c|}
\hline & & SPA & & SSL & \\
\hline $\begin{array}{l}\text { Item } \\
\text { (IV) }\end{array}$ & Medida preventiva & $\mathrm{n}$ & $\%$ & n & $\%$ \\
\hline 28 & $\begin{array}{l}\text { En caso de exposición a aerosoles } \\
\text { químicos se dispone de los EPI } \\
\text { respiratorios necesarios }\end{array}$ & 156 & 98,7 & 116 & 91,4 \\
\hline 29 & $\begin{array}{l}\text { En caso de ser necesario disponen } \\
\text { de guantes con protección frente a } \\
\text { microorganismos }\end{array}$ & 192 & 92,7 & 72 & 81,9 \\
\hline 30 & $\begin{array}{l}\text { El personal que realiza la limpieza } \\
\text { cuenta con la protección necesaria } \\
\text { (mascarilla auto filtrante FFP2 } \\
\text { o superior y guantes frente a } \\
\text { microorganismos) }\end{array}$ & 277 & 92,1 & 267 & 83,1 \\
\hline \multicolumn{2}{|c|}{ Total de empresas visitadas } & 361 & 100.0 & 298 & 100.0 \\
\hline
\end{tabular}

a Ítem del cuestionario a valorar. SPA: Servicio de Prevención de Riesgos Laborales SSL: Servicio de Salud Laboral del ISPLN

La tabla 3 muestra que en la casi generalidad de empresas industriales se han aplicado medidas para garantizar la distancia interpersonal de seguridad (IV7, IV9, IV10, IV11). Las medidas técnicas se han acompañado de medidas organizativas para limitar el número de personas (IV2, IV3) y las distancias (IV8). Los aspectos psicosociales derivados de la pandemia se han abordado con menor frecuencia (IV5). La disponibilidad de productos y programación de la limpieza de lugares y equipos de trabajo está establecida en más del 80\% de las empresas (IV13, IV14, IV15, IV16, IV19). Sus instrucciones por escrito no constan con tanta frecuencia. La disponibilidad de recursos de higiene personal (IV17, IV18, IV20) es muy generalizada, excepto la presencia de papeleras adecuadas. La disponibilidad de EPI respiratorio y otros equipos frente a riesgo biológico existe en más del $80 \%$ de las empresas (IV24 a IV29). Respecto a la información y formación (IV21, IV22, IV23), la formación teórico práctica se ha dado y la cartelería está presente entre el 40 y el $60 \%$ de las empresas según el personal técnico del SSL, mientras se aplica en casi el $90 \%$ de las mismas según el personal de los SPA.

Las medidas han considerado al personal de las contratas (IV4) y de limpieza (IV30). El cuestionario de industria se interesa por la ventilación de los lugares de trabajo (IV12). Por último, el SSL considera que en el $45 \%$ de las empresas, no se ha dado la necesaria participación a la hora de aplicar las medidas preventivas (IV1), mientras los SPA reducen esta frecuencia al 15\% de las empresas.

Dada la escasa comparabilidad del trabajo industrial y de construcción, con cautela diremos que la industria muestra mayor capacidad para aplicar las medidas preventivas y protectoras frente a la Covid-19 que la construcción (distancias, limpieza, recursos para higiene personal). La dotación de EPI y otras protecciones parece adecuada, mientras la cartelería informativa y el componente práctico de la formación presenta deficiencias en ambos sectores. 


\section{Discusión}

Estos resultados han puesto de manifiesto las debilidades en la consecución de espacios seguros en el trabajo en los sectores de la construcción e industria durante la fase de desescalada en el contexto de la pandemia de la Covid-19. Respecto a la realidad preventiva en las empresas, los resultados muestran que es necesario consolidar la vigilancia de la aplicación y mantenimiento de las medidas preventivas frente a la Covid-19. En este sentido, y bajo criterio de jerarquía de medidas preventivas, los resultados indican que es preciso incrementar la información sobre la enfermedad y su prevención para evitar la presencia de personas enfermas, y realizar formación práctica sobre uso de EPI y otros protectores. También es necesario reforzar la atención a los riesgos asociados a los cambios habidos en la organización del trabajo como: descenso de la plantilla por incapacidad laboral, aumentos de producción en algunas actividades, proliferación del teletrabajo, incertidumbre, entre otras. La información aportada desde las personas trabajadoras para comprender las características de la exposición, y sobre cómo aplicar las medidas preventivas, es insustituible. En una primera aproximación la representación sindical y empresarial ha valorado positivamente la experiencia de acompañamiento realizada desde el SSL con el PV.

El volumen de empresas industriales y obras de construcción visitadas, sin sesgo conocido en su selección en un corto periodo de tiempo, aportan una visión general de la situación y permite impulsar la creación de espacios seguros de trabajo. El contar con la coordinación ejecutiva de los SPA, posibilitó disponer de un número importante de personal técnico capacitado para visitar empresas, recoger datos, proponer medidas de control del riesgo y vigilar su cumplimiento. Esa coordinación ejecutiva ha sido, hasta donde hemos comprobado, la primera experiencia de este tipo en el territorio español.

Entre las limitaciones se debe considerar, en primer lugar, que, pese a que las dos muestras de empresas y obras estudiadas no aportan datos representativos, al no estar seleccionadas al azar, su amplitud y la aparente falta de aplicación de criterios de selección da solidez a los mismos. Los errores en la base de datos del Registro de Cotización de Empresas de la Tesorería General de la Seguridad Social a fecha 26 de abril -utilizada para la identificación de las mismas- y la propia situación de descenso de la actividad económica, explican el encontrarse con empresas ilocalizables o cerradas.

De igual forma, la marcada diferencia de percepción en la identificación de los peligros y la realidad de la gestión preventiva de los SPA y el personal de la Administración, no es un hecho nuevo(6). En este estudio, la falta de homogeneidad de resultados del personal técnico de los SPA y del SSL, puede asociarse a la no formación presencial del personal técnico para el PV, a la distinta experiencia de asesoramiento a las empresas, y a una hipotética diferencia de volumen de trabajadores de las empresas visitadas entre ambos tipos de profesionales, entre otros factores. 
En tercer lugar, puede constatarse cierta falta de homogeneidad técnica entre ambos cuestionarios: protección respiratoria, dérmica, participación, riesgos psicosociales, entre otros. Se debe a su formulación por dos equipos distintos, al uso de distintas fuentes y documentos científico - técnicos en acelerado cambio según fecha, a la adaptación de los cuestionarios iniciales tras las fases piloto, y a la distinta realidad de los centros de trabajo de industria y construcción.

Por último, también debe tenerse presente que el registro oficial SERPA(7), de ámbito estatal, no permite conocer las empresas atendidas por cada uno de los SPA lo cual debiera ser resuelto normativamente.

Esta pandemia pone en evidencia la relevancia del papel a jugar por las Administraciones Públicas con intervenciones de salud pública en los lugares de trabajo, en aras a proteger a la población trabajadora y a la población general(8). La coordinación ejecutiva de los SPA ejercida por el SSL (ISPLN), ha permitido colaborar eficazmente en la atención de las necesidades preventivas de las empresas en la desescalada de los sectores de industria y construcción en la pandemia de Covid-19. Sería conveniente realizar la valoración de la experiencia con las direcciones y el personal técnico de los SPA.

La especial caracterización de las medidas preventivas, emanadas bajo la autoridad sanitaria, requiere resolver con cierta urgencia la ausencia del desarrollo reglamentario de la tipificación de las sanciones establecidas en Ley General de Salud Pública. Esta carencia impide la imposición eficiente de sanciones a SPRL y a empresas incumplidoras.

\section{Agradecimientos}

Expresamos nuestro agradecimiento a todo el personal técnico que ha participado en el trabajo de campo, verdadero artífice de la disposición de los datos presentados en este artículo.

\section{Bibliografía}

1. Koh D. Occupational risks for COVID-19 infection. Occupational Medicine. 2020;70:3-5. Disponible en: https://bit.ly/3i3icGr

2. Dyal JW, Grant MP, Broadwater K, et al. COVID-19 among workers in meat and poultry processing facilities - 19 States, April 2020. MMWR Morb Mortal Wkly Rep. 2020;69:557-561. doi:10.15585/mmwr.mm6918e3. pmid: 32379731

3. ISPLN. Recomendaciones preventivas frente al covid-19 en el sector industrial. Disponible en: https://bit.ly/3mcOAZh

4. ISPLN. Recomendaciones preventivas frente al covid-19 en el sector construcción. Disponible en: https://bit.ly/322TkYK

5. Decreto-ley Foral 3/2020, de 15 de abril, por el que se aprueban medidas urgentes para responder al impacto generado por la crisis sanitaria del coronavirus (CO- 
VID-19). BON N. ${ }^{\circ} 80$ - 17/04/2020. Disponible en: http://www.lexnavarra.navarra. es/detalle.asp? $r=52597$

6. Decreto-ley Foral por el que se aprueban medidas urgentes para responder al impacto generado por la crisis sanitaria del coronavirus (COVID-19). DLF 3/2020 de 15 de abril 2020. BOE núm. 124, de 4 de mayo de 2020, páginas 31128 a 31142. Disponible en: https://bit.ly/2YWpnqQ

7. Institut Català de Seguretat i Salut Laboral. Gestió de la prevencion de riscos laborals a les empreses de Catalunya, 2016. [Fecha de última consulta: 26 de mayo de 2020]. Disponible en: https://bit.ly/3dvi4vy

8. Orden Foral 276/2014, de 9 de septiembre, de la consejera de economía, hacienda, industria y empleo por la que se crea el registro de entidades especializadas para actuar como servicios de prevención ajenos en Navarra. Disponible en: https://bit.ly/31hvXLH

9. Baker MG, Peckham TK, Seixas NS. Estimating the burden of United States workers exposed to infection or disease: A key factor in containing risk of COVID-19 infection. PLoS ONE. 2020;15(4):e0232452. Disponible en: https://bit.ly/2VcgloQ 